\title{
Impact of residual disease as a prognostic factor for survival in women with advanced epithelial ovarian cancer after primary surgery (Protocol)
}

Bryant A, Hiu S, Kunonga P, Gajjar K, Craig D, Vale L, Winter-Roach BA, Elattar A, Naik R

Bryant A, Hiu S, Kunonga P, Gajjar K, Craig D, Vale L, Winter-Roach BA, Elattar A, Naik R.

Impact of residual disease as a prognostic factor for survival in women with advanced epithelial ovarian cancer after primary surgery (Protocol).

Cochrane Database of Systematic Reviews 2021, Issue 9. Art. No.: CD015048.

DOI: 10.1002/14651858.CD015048. 
TABLE OF CONTENTS

HEADER 1

ABSTRACT

BACKGROUND

OBJECTIVES

METHODS

ACKNOWLEDGEMENTS

REFERENCES

APPENDICES

CONTRIBUTIONS OF AUTHORS

DECLARATIONS OF INTEREST

SOURCES OF SUPPORT 
[Prognosis Protocol]

\section{Impact of residual disease as a prognostic factor for survival in women with advanced epithelial ovarian cancer after primary surgery}

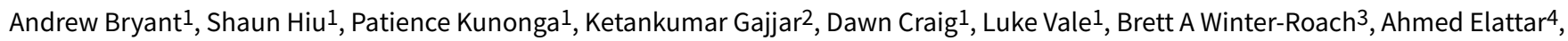
Raj Naik 5

1Population Health Sciences Institute, Newcastle University, Newcastle upon Tyne, UK. 2Department of Gynaecological Oncology, 1st Floor Maternity Unit, City Hospital Campus, Nottingham, UK. ${ }^{3}$ The Department of Surgery, Christie Hospital NHS Foundation Trust, Manchester, UK. ${ }^{4}$ City Hospital \& Birmingham Treatment Centre, Birmingham, UK. 5 Gynaecological Oncology, Northern Gynaecological Oncology Centre, Gateshead, UK

Contact address: Andrew Bryant, Andy.Bryant@ncl.ac.uk.

Editorial group: Cochrane Gynaecological, Neuro-oncology and Orphan Cancer Group.

Publication status and date: New, published in Issue 9, 2021.

Citation: Bryant A, Hiu S, Kunonga P, Gajjar K, Craig D, Vale L, Winter-Roach BA, Elattar A, Naik R. Impact of residual disease as a prognostic factor for survival in women with advanced epithelial ovarian cancer after primary surgery (Protocol). Cochrane Database of Systematic Reviews 2021, Issue 9. Art. No.: CD015048. DOI: 10.1002/14651858.CD015048.

Copyright $\odot 2021$ The Cochrane Collaboration. Published by John Wiley \& Sons, Ltd.

\section{A B S T R A C T}

\section{Objectives}

This is a protocol for a Cochrane Review (prognosis). The objectives are as follows:

To assess the impact of residual disease after primary surgery on survival outcomes. In separate analyses, primary surgery will include both upfront debulking surgery followed by adjuvant chemotherapy (UDS) and neoadjuvant chemotherapy followed by interval debulking surgery (IDS). We will consider each residual disease threshold as a separate prognostic factor.

\section{Investigation of sources of heterogeneity}

We will examine differences between FIGO stages III and IV in different thresholds of residual disease after primary surgery.

We will consider factors such as age, grade, length of follow-up, type and experience of surgeon and type of surgery in the interpretation of any heterogeneity. We will also perform sensitivity analyses to distinguish between studies that include $0 \mathrm{~cm}$ in residual disease (RD) categories of $<1 \mathrm{~cm}$ and those that do not. This may be applicable to comparisons involving $\mathrm{RD}<1 \mathrm{~cm}$ with the exception of RD $<1 \mathrm{~cm}$ versus $\mathrm{RD}=0 \mathrm{~cm}$.

We will evaluate women undergoing UDS and IDS in separate analyses. 


\section{B A C K G R O U N D}

\section{Description of the health condition and context}

Ovarian cancer is the seventh most common cancer among women and a leading cause of death in women with gynaecological malignancies (GLOBOCAN 2018). Globally, there are approaching 300,000 new cases per year, with approximately 6.6 new cases per 100,000 women per year. A woman's cumulative risk of developing ovarian cancer by the age of 75 years is $0.72 \%: 0.52 \%$ in low-income countries and $0.92 \%$ in high-income countries (GLOBOCAN 2018). Ovarian cancer is rare in women under 40 years of age and most cancers in this age group are germ cell tumours. Above age 40 , more than $90 \%$ are epithelial tumours and the risk increases with age (Kurman 2014; Webb 2017).

Ovarian cancer is best regarded as a peritoneal malignancy. The current understanding on the pathogenesis of epithelial ovarian cancer (EOC) recognises two pathways and two clinical groupings, classified as Type 1 and Type 2. Type 1 tumours comprise low-grade serous, low-grade endometrioid, clear-cell and mucinous carcinomas, and Brenner tumours. Type 2 tumours comprise the high-grade serous and endometrioid carcinomas, mixed mullerian tumours and undifferentiated carcinomas. Type 2 tumours are more common and are thought to have their origin within the fallopian tube (Perets 2016). They are associated with the BRCA (breast cancer gene) germline and somatic mutations, and histopathologically identified with aberrant p53 expression and other characteristic immunohistochemical features (Kurman 2010; Kurman 2011).

The extent of dissemination of the disease is described using the International Federation of Gynecology and Obstetrics (FIGO) staging system; stage I disease is confined to the ovaries; stage II disease is confined to the true pelvis, stage III disease is an abdominal disease where there is spread to the lining (peritoneum) of the abdominal cavity outside the pelvis or regional lymph node spread; whilst stage IV disease is outside the abdomen or parenchymatous metastases, e.g. disease with spread to distant organs such as the chest or liver (Berek 2018). Thirty per cent of women with ovarian cancer present with early stage disease, whilst $70 \%$ have advanced stage at presentation (Torre 2018).In Europe, just over a third of women with ovarian cancer are alive five years after diagnosis (EUROCARE 2015), largely because most women with ovarian cancer are diagnosed when the cancer is already at an advanced stage (Jemal 2017).

The majority of women who present with symptoms of ovarian cancer already have advanced disease. Symptoms include progressive feelings of: abdominal distension, bloating, indigestion, urinary frequency, urgency, early satiety, weight loss, reduced appetite, abdominal and pelvic pain and, less commonly, vaginal bleeding (Shafi 2018).

\section{Description of the surgical interventions and residual disease as a prognostic factor}

Surgery and chemotherapy are the mainstay of treatment for the $70 \%$ of women who present with advanced disease (FIGO stage III/ IV) when surgery alone cannot be curative (Fader 2007; Torre 2018).

Appropriate initial investigations usually include ultrasonography, tumour markers, and a CT scan if malignancy is suggested by tumour markers and ultrasound. If required, an ultrasound-guided biopsy of metastatic spread is carried out to obtain histological diagnosis (Shafi 2018).

Traditionally,upfront debulking surgery (UDS) is performed to achieve optimal cytoreduction, as the amount of residual tumour is one of the most important prognostic factors for survival of epithelial ovarian cancer (Bristow 2002; Chang 2013; du Bois 2009; Griffiths 1975; Hoskins 1994; Wimberger 2010).Platinumbased chemotherapy is the standard of care, in combination with debulking surgery (Colombo 2019; National Comprehensive Cancer Network 2020).

Chemotherapy followed by interval debulking surgery (IDS) is an alternative and more recent primary treatment option for women diagnosed with advanced ovarian cancer. A Cochrane Review comprehensively reviewed the evidence in this area, which comprised five randomised controlled trials (RCTs) (Coleridge 2021). The review assessed survival, quality of life and morbidity outcomes in trials that compared upfront primary and interval debulking surgery. The five trials included two large, welldocumented RCTs (CHORUS (Kehoe 2015) and EORTC 55971 (Vergote 2010)) which reported no significant difference in survival between IDS compared with UDS. It was suggested that IDS may have better overall survival in stage IV disease, whereas women with FIGO stage IIIC disease with extrapelvic metastases smaller than $5 \mathrm{~cm}$ may have better progression-free survival after upfront debulking (Vergote 2018). The selection of women with advanced ovarian cancer for UDS or IDS remains controversial (Vergote 2013). An investigation of maximum effort cytoreductive surgery during the initial treatment of epithelial ovarian cancer comparing UDS versus IDS is being investigated in the TRUST trial (Trial of Radical Upfront Surgical Therapy in advanced ovarian cancer (ENGOT ov33/ AGO-OVAR OP7)), and we await the results in 2024 (Reuss 2019).

The terms cytoreductive and debulking surgery are often used interchangeably to indicate surgical efforts aimed at removing the bulk of the tumour. Complete macroscopic resection is achieved when there is no visible tumour left at the end of surgery. Previously, the term 'optimal cytoreduction' had been variably defined as referring to a maximal diameter of residual tumour left behind after surgery measuring 0 to $2 \mathrm{~cm}$, and in 1994 the Gynaecologic Oncology Group (GOG) defined optimal cytoreduction as having residual disease $<1 \mathrm{~cm}$ (Hoskins 1994). However, in 2010 the Gynaecological Cancer Inter-Group defined 'optimal' as having no visible residual tumour nodules, i.e. complete macroscopic resection (since microscopic disease remains in the majority of patients) (Stuart 2011), which has been shown to result in better survival than near-optimal $(<1 \mathrm{~cm})$ and suboptimal cytoreduction $(>1 \mathrm{~cm})$ and to be a better predictor of survival (Bookman 2009; Chang 2013; du Bois 2009; Sørensen 2019; Wimberger 2010). While there is now less controversy about the prognostic importance of maximum cytoreduction, there remains divided opinion about the effects of any remaining residual disease after UDS or IDS and about what attempts should be made for maximal efforts at debulking. All women would potentially do better if there is no residual disease after surgery, and obviously no surgeon sets out for suboptimal cytoreduction from the onset. However, different philosophies are evident within the surgical community and there are also other important considerations, such as surgical skills, training, the woman's fitness for more radical treatment, morbidity, mortality and quality of life. The questions 
about UDS in ovarian cancer that appear to have become more important and relevant over the last 10 years of practice as other evidence has emerged relate to the timing of maximal surgical effort (still within initial treatment phase), and to consideration of whether there are some histological subtypes which may have better outcomes with UDS. In this review we only consider the epithelial subtype of ovarian cancer, since it comprises $90 \%$ of histological subtypes.

Complete macroscopic resection appears to be associated with the best chance of prolonged survival (Bookman 2009). An attempt to achieve complete cytoreduction is the recommended standard for cytoreductive surgery for advanced ovarian cancer, as advised by the British Gynaecological Cancer Society (BGCS) (BGCS 2017), European Society of Medical Oncology (ESMO) and European Society of Gynaecological Oncology (ESGO) (Colombo 2019), and the National Comprehensive Cancer Network (NCCN) (National Comprehensive Cancer Network 2020).

A Cochrane Review assessed the role of a further attempt at cytoreductive effort after suboptimal primary surgery (Tangjitgamol 2016). The results from three studies in the review found that a further attempt at debulking surgery after chemotherapy in first line treatment was only of benefit to those who had not had their initial surgery performed by a gynaecological oncologist (Redman 1994; Rose 2004; Van der Burg 1995).

Over the last few decades, efforts have been made to increase complete resection rates. It has been shown that surgery performed by gynaecologists with training in gynaecological oncology, by high volume surgeons and high volume centres, is associated with increased likelihood of no macroscopic disease (Bristow 2009; Greggi 2016; Woo 2012).

There is a widespread belief that tumour biology has a significant role to play in ovarian cancer outcomes. The relationship between surgical outcome and tumour biology is complex and remains unclear. The biological rationale behind the benefit of surgical cytoreduction is that removal of certain ovarian cancer tumour cells will create a supportive microenvironment to enhance chemotherapy effect (Covens 2000; Napoletano 2010). Whether it is the intrinsic biological behaviour of the tumour or the surgeon's ability to cytoreduce that determines optimal cytoreduction is not well studied. However, among the relevant prognostic factors, the extent of surgery and consequent residual disease are the most important prognostic factors. The extent of surgical effort (standard versus extensive surgery) to achieve complete resection and its impact on survival is not fully understood, as determined by a previous Cochrane Review (Ang 2011).

Within the advanced ovarian cancer group, women with stage IV ovarian cancer represent a heterogeneous group with extraperitoneal metastases. While it has been shown in a previously published guideline that complete macroscopic resection confers the best chance of prolonged survival (Vergote 2016), the data are not as convincing for stage IV ovarian cancer. The presence of microscopic disease in the extraperitoneal locations has not been assessed and can potentially be even more frequent. While some stage IV diseases could be amenable to macroscopic resection (isolated splenic parenchymal lesion or resectable liver metastasis), others could be difficult (extensive mediastinal, axillary, or supraclavicular nodes or multiple, unresectable hepatic metastases). Therefore, it is worth investigating the impact of residual disease in stage IV cancers, and in particular in relation to extra-peritoneal residual disease (thoracic, mediastinum, groin, axilla, neck). The EORTC55971 trial confirmed that neoadjuvant chemotherapy results in superior survival compared with primary debulking surgery in the management of women with stage IV disease (Vergote 2010). However, there is a need for further investigation into the impact of residual disease on survival between the UDS and IDS subgroups.

This review sets out to determine the prognostic impact of residual disease on survival rates in women with advanced epithelial ovarian cancer. There are no universally established patient selection criteria, but certain baseline characteristics are important when investigating the impact of residual disease on prognosis. These include age, nutritional status, FIGO stage, comorbidities, ASA score (American Society of Anaesthesiologists' (ASA) classification of Physical Health), ECOG (Eastern Cooperative Oncology Group) performance status (score of symptom and functional status with respect to ambulatory status and need for care), BRCA status, presence of ascites on preoperative imaging and histological grade (du Bois 2009). To date, there are no specific predictive models for surgical success that are clinically useful, and the majority of previous studies have limitations in design that make their interpretation difficult (Borley 2012).

If the surgical outcome and prognosis are to be determined by tumour biology alone, the residual disease after surgery may have little influence on overall survival. However, tumour biology and the extent of disease may influence the likelihood of achieving no macroscopic disease after surgery (Colombo 2019). The extent of residual disease and prognosis could be influenced by the extent of disease measured intraoperatively by the peritoneal cancer index (PCI) score, surgical complexity score (SCS) (Elzarkaa 2018), type and extent of surgery (Aletti 2007), characteristics of the surgical team (gynaecological oncologist in a specialist centre with high volume of cases) (Bristow 2009) and presence of ascites during surgery (du Bois 2009).

\section{Why it is important to do this review}

A greater understanding of the biology of ovarian cancer variants, especially with respect to BRCA gene mutations, has led to more sophisticated treatment regimens. These include the emergence of tailored adjuvant and maintenance chemotherapeutic options for women with BRCA somatic and germline mutations, and greater options for the chemotherapeutic approach to recurrent disease (Colombo 2019).

While the place of surgery in the context of treatment of ovarian cancer is well established, the distinctive biological phenotypes (e.g. type and grade of disease, extent of disease) should be anticipated to lead to some heterogeneity in the level of benefit derived from maximal surgical effort. There may be a greater willingness to rely on UDS for women with known subtypes of disease, such as low-grade serous cancer, that are known to be less chemo-responsive (Grabowski 2016). UDS for highly chemo-responsive disease has also been questioned by a growing acceptance of the non-inferiority of interval debulking surgery (Coleridge 2021). The current position in many settings in the UK and elsewhere is to reserve UDS in advanced disease for those women who have a good performance status, and in whom it is anticipated that complete or near macroscopic resection 
(cytoreduction) can be achieved. Performance status is relevant in consideration of UDS. Though true advocates of UDS remain, many clinicians recognise that women presenting with poor performance status are likely to be too frail to undergo a UDS without significant comorbidity. In such a situation, clinical optimisation and initiation of treatment with chemotherapy is preferable with a possible benefit of reduced morbidity by reduction in disease burden with chemotherapy (Kumar 2017).

There is consensus that the surgery performed during the initial treatment of ovarian cancer, whether UDS or IDS, should aim to remove all macroscopic disease. The need for clarity on the location (cancer centre or unit) and timing from diagnosis of first look surgery (intensive staging and cytoreductive surgery) for advanced ovarian cancer has never been more relevant. Women, clinicians and commissioners of specialist cancer services need to know what the overall benefit of cytoreductive surgery for ovarian cancer is, and to determine if there are subgroups of women for whom this intervention is of greater value. Given the diversity recognised within the overall group of women with advanced-stage ovarian cancer, it is anticipated that an ethos of individualised surgical planning, whilst recognising overarching principles, would be appropriate. One recent study compared operative approaches/ philosophies, where an ultra-radical approach to surgery was introduced at a population level, but this was not a controlled study (Falconer 2020). In this population-based cohort study, all women with suspected EOC in a region of Stockholm in two national cancer registries were selected in two three-year cohorts, based on year of diagnosis (before (cohort 1) or after (cohort 2) change in surgical treatment algorithm) and followed for at least three years. The study reported five-year overall survival in non-surgically and surgically treated women. A similar study into system reorganisation that uses either a controlled before-andafter component or interrupted time series design would be able to look at the impact of any centralisation of more radical surgery on survival.

Although the size of residual tumour mass after surgery has been shown to be an important prognostic factor for advanced ovarian cancer, there is limited evidence to support the conclusion that the surgical procedure is directly responsible for the superior outcome associated with less residual disease (Girling 1996; Hunter 1992).

Whether optimal cytoreduction is more feasible in women with biologically less aggressive tumours is a subject for continual debate. Tumour biology is not thought to be the only factor affecting prognosis (Sørensen 2019), and its impact seems to be partially overruled by the extent of residual disease, i.e. whether complete and optimal cytoreduction was performed (du Bois 2009). It has also been suggested that further evaluation of biological factors may help select women who are most likely to benefit from UDS (du Bois 2009; Markar 2016). It has been suggested that women whose cancer is cytoreduced to macroscopic and near-macroscopic disease at UDS may have superimposable progression-free survival, meaning that women with high tumour load, completely resected at the time of surgery, may have micro/ macroscopic unrecognised residual disease (Fagotti 2020). In this review, we will analyse UDS and IDS separately because the former achieving cytoreduction to $<1 \mathrm{~cm}$ may be equivalent to IDS achieving cytoreduction to macroscopic $(0 \mathrm{~cm})$ disease, in the context of debulking.
The aim of this review is to investigate the effects of residual disease in women who received upfront or interval cytoreductive surgery for advanced epithelial ovarian cancer. This review should help to determine the impact residual disease after surgery has on survival.

\section{O B J E C T I VES}

To assess the impact of residual disease after primary surgery on survival outcomes. In separate analyses, primary surgery will include both upfront debulking surgery followed by adjuvant chemotherapy (UDS) and neoadjuvant chemotherapy followed by interval debulking surgery (IDS). We will consider each residual disease threshold as a separate prognostic factor.

\section{Investigation of sources of heterogeneity}

We will examine differences between FIGO stages III and IV in different thresholds of residual disease after primary surgery.

We will consider factors such as age, grade, length of follow-up, type and experience of surgeon and type of surgery in the interpretation of any heterogeneity. We will also perform sensitivity analyses to distinguish between studies that include $0 \mathrm{~cm}$ in residual disease (RD) categories of $<1 \mathrm{~cm}$ and those that do not. This may be applicable to comparisons involving $\mathrm{RD}<1 \mathrm{~cm}$ with the exception of $R D<1 \mathrm{~cm}$ versus $R D=0 \mathrm{~cm}$.

We will evaluate women undergoing UDS and IDS in separate analyses.

\section{METHODS}

\section{Criteria for considering studies for this review \\ Types of studies}

We will include data from RCTs, prospective and retrospective cohort studies and unselected case series of 100 or more women that include concurrent comparison of different RD thresholds after primary surgical intervention. Any data collected from RCTs will be retrospective and taken from trials that randomised groups of women to various chemotherapy protocols after UDS or IDS debulking surgery. We will categorise the surgical outcome as macroscopic, optimal and suboptimal debulking, based on the maximum size of postoperative residual disease.

In order to minimise bias, we will only include studies of multivariate Cox regression models that use sensible adjustment factors associated with survival in women with advanced EOC (e.g. age, stage, grade, extent of disease at diagnosis). We will exclude studies that only report unadjusted results. To assess the adequacy of adjustment factors used in multivariate Cox models, we will use the 'adjustment for other prognostic factors' and 'statistical analysis and reporting' domains of the quality in prognosis studies (QUIPS) tool (Riley 2019). Therefore, in theory, only one other factor would need to be adjusted for for the study to meet inclusion in the review, but such studies would be judged as being at high risk of bias in these domains.

We will exclude case-control studies, studies that do not have concurrent comparison groups and case series of fewer than 100 women. This is in an attempt to optimise the quality of the review, as poor study designs will introduce additional forms of bias. The inclusion of adequately-sized studies, although pragmatic, may 
also provide more reliable estimates due to restricting results to those reporting multiple adjustments in statistical models.

\section{Types of participants}

We will include adult women (over 18 years of age) with surgicallystaged advanced epithelial ovarian cancer (FIGO stages III and IV) who had confirmed histological diagnoses. We will exclude women with other concurrent malignancies.

\section{Details of prognostic factor}

The surgical intervention for which we will assess the resulting prognostic factor is primary debulking surgery (upfront and interval debulking).

We will include studies that include all RD thresholds after surgery but will define optimal RD as surgery leading to residual tumours with a maximum diameter of any threshold up to $1 \mathrm{~cm}$. The main $\mathrm{RD}$ thresholds of interest will be microscopic RD (labelled as no macroscopic RD); RD < $1 \mathrm{~cm}$, categorised as 'near-optimal'; and RD > $1 \mathrm{~cm}$, categorised as 'suboptimal'. However, we will include studies reporting any size of RD. We will note details of any women who have primary surgery that results in RD which does not meet the criteria specified in the study as 'optimal', namely not categorised as 'macroscopic' or 'near-optimal' cytoreduction.

We will apply the above RD thresholds to both UDS (upfront debulking surgery followed by platinum based chemotherapy) and IDS (platinum chemotherapy followed by interval debulking surgery) settings.

- Macroscopic cytoreduction (RD $0 \mathrm{~cm})$

- Near-optimal cytoreduction $(\mathrm{RD}<1 \mathrm{~cm})$

- Suboptimal cytoreduction $(\mathrm{RD}>1 \mathrm{~cm})$

\section{Types of outcome measures}

- Overall survival: survival until death from any cause. We will assess survival from the time at which women were enrolled in the study.

- Progression-free survival

We will extract survival estimates as time-to-event data from an adjusted multivariate Cox model (as outlined above in Types of studies). This is the most appropriate way to analyse these outcomes as it accounts for any loss to follow-up and will correctly allow for censoring.

\section{Search methods for identification of studies}

We will seek papers in all languages and translate them if necessary.

\section{Electronic searches}

We will search the following electronic databases.

- Cochrane Gynaecological Cancer Collaborative Review Group Trials Register

- Cochrane Central Register of Controlled Trials (CENTRAL) (The Cochrane Library latest issue)

- MEDLINE

- Embase
The MEDLINE search strategy is based on terms related to the review topic and is presented in Appendix 1. For databases other than MEDLINE, we will adapt the search strategy accordingly. We will search the databases from 1950 to present.

For all relevant articles found, we will identify them on PubMed and use the 'related articles' feature to carry out a further search for newly-published articles.

\section{Searching other resources}

\section{Unpublished and grey literature}

We will search Metaregister, Physicians Data Query, www.controlled-trials.com/rct, www.clinicaltrials.gov and www.cancer.gov/clinicaltrials for ongoing trials.

\section{Handsearching}

We will check the citation lists of relevant publications, abstracts of scientific meetings and included studies through handsearching, and we will contact experts in the field to identify further reports of trials. We will handsearch reports of conferences from the following sources.

- Gynecologic Oncology (Annual Meeting of the American Society of Gynecologic Oncologists)

- International Journal of Gynecological Cancer (Annual Meeting of the International Gynecologic Cancer Society)

- British Journal of Cancer

- British Cancer Research Meeting

- Annual Meeting of European Society of Medical Oncology (ESMO)

- Annual Meeting of the American Society of Clinical Oncology (ASCO)

\section{Correspondence}

We will contact authors of relevant trials to ask if they know of further data, which may or may be published.

\section{Data collection and analysis}

\section{Selection of studies}

We will download all titles and abstracts retrieved by electronic searching to the reference management database Endnote. After removing duplicates, three review authors $(\mathrm{AB}, \mathrm{PK}, \mathrm{SH})$ will examine the remaining references independently. We will exclude those studies which clearly do not meet the inclusion criteria and obtain copies of the full text of potentially relevant references. Three review authors (AB, PK, SH) will assess the eligibility of retrieved papers independently. We will resolve disagreements by discussion between the three review authors or, if necessary, by appeal to a fourth review author (RN, KG). We will document reasons for exclusion.

\section{Data extraction and management}

For included studies, we will extract items relevant to prognostic factor studies, derived from the checklist for critical appraisal and data extraction for systematic reviews of prediction modelling studies (CHARMS) (Moons 2014). This will include data on the following. 
- Author, year of publication and journal citation (including language)

- Country

- Setting

- Inclusion and exclusion criteria

- Study design, methodology

- Study population:

* total number enrolled in each group;

* participant characteristics;

* age;

* comorbidities.

- Ovarian cancer details at diagnosis:

* FIGO stage (III or IV);

* histological cell type;

* preoperative tumour volume;

* ascites (large or small volume);

* tumour grade;

* extent of disease.

- Surgical intervention details:

* details of primary optimal cytoreductive surgery; $\square$ Upfront and interval debulking settings

* details of platinum based chemotherapy; $\square$ dose, number of chemotherapy cycles before and after surgery

* type of surgeon (gynaecological oncologist, gynaecologist, general surgeon);

* experience of surgeon;

* type of surgery (ultra-radical or standard).

- Details of prognostic factor:

* details of residual disease;

* definition of optimal and suboptimal residual disease in study;

* covariates included in multivariate Cox models for survival that include residual diease.

- Risk of bias in study (see Assessent of risk of bias in included studies)

- Duration of follow-up

- Outcomes (see Types of outcome measures)

For time-to-event data (survival and progression-free survival), we will extract the $\log$ of the hazard ratio $(\log (\mathrm{HR}))$ and its standard error from study reports; if the study does not report these, we will not attempt to estimate the log (HR) and its standard error using the methods of Parmar 1998, as we are only including adjusted analyses.

We will note the time points at which outcomes are collected and reported.

Three review authors (AB, PB, SH) will independently extract data using a data collection form specially designed for the review. We will resolve differences between review authors by discussion or by appeal to a fourth review author (KG), if necessary.

\section{Assessment of risk of bias in included studies}

Three review authors will independently extract data and assess risk of bias. We will extract the data using the CHARMS-PF (checklist for critical appraisal and data extraction for systematic reviews prognostic factor studies; Riley 2019). We will assess risk of bias (and appraise quality) in the prognostic assessment of residual diease in included studies using the quality in prognosis studies (QUIPS) tool (Appendix 2). QUIPS is a tool designed to assess risk of bias in prognostic factor studies (Riley 2019). It assesses bias across the following six domains using intermediate signalling questions to aid the decision making process.

1. Participant selection

2. Study attrition

3. Prognostic factor measurement

4. Outcome measurement

5. Adjustment for other prognostic factors

6. Statistical analysis and reporting

In addition, we will give consideration to the applicability of the study for four of the domains, as reported in other tools (Whiting 2011; Wolff 2019). We will judge risk of bias and concerns regarding applicability using the tools shown in Appendix 2. The questions regarding applicability will include the following.

- Domain 1: participant selection. Are there concerns that the included women do not match the review question?

- Domain 3: prognostic factor measurement. Are there concerns that residual disease, the way that it is measured, or the way that it is interpreted, differ from the review question?

- Domain 4: outcome measurement. Are there concerns that outcome does not match the review question or that follow-up was not of sufficient duration?

- Domain 5: adjustment for other prognostic factors. Did the prognostic factors adjusted for match the review question?

Three review authors (AB, PK, SH) will apply the risk of bias tool independently and resolve differences by discussion or by appeal to a fourth review author (KG). We will present results in a risk of bias summary table. We will interpret results of meta-analyses in light of the findings with respect to risk of bias.

\section{Measures of treatment effect}

For time-to-event data (overall and progression-free survival), we will use the adjusted hazard ratio (HR). We will not use unadjusted results, as outlined above in Types of studies.

\section{Dealing with missing data}

We will not impute missing outcome data for any of the outcomes.

\section{Assessment of heterogeneity}

We will assess heterogeneity between studies by visual inspection of forest plots, by estimation of the percentage of heterogeneity between trials which cannot be ascribed to sampling variation (Higgins 2003), by a formal statistical test of the significance of the heterogeneity (Deeks 2001), and, if possible, by subgroup analyses (see 'Subgroup analysis and investigation of heterogeneity'). If there is evidence of substantial heterogeneity, we will investigate and report the possible reasons for this.

\section{Assessment of reporting biases}

We will examine the symmetry of funnel plots corresponding to meta-analyses of overall survival to assess the potential for small 
study effects in analyses containing 10 or more studies. We will test for asymmetry where evidence of asymmetry may be an indicator of publication bias (Debray 2018; Sterne 2011).

\section{Data synthesis}

If sufficient clinically similar studies are available, we will pool their adjusted results in meta-analyses. We will report results by FIGO stage (see 'Subgroup analysis and investigation of heterogeneity').

- For time-to-event data, we will pool hazard ratios (HRs) using the generic inverse variance facility of Review Manager 2020.

- We will use random-effects models with inverse variance weighting for all meta-analyses (DerSimonian 1986).

- We will report analyses separately for women who receive upfront and interval debulking surgery.

\section{Subgroup analysis and investigation of heterogeneity}

We will consider factors such as age, grade, length of follow-up, type and experience of surgeon and type of surgery in the interpretation of any heterogeneity.

We will perform subgroup analysis grouping studies by women with FIGO stage III versus stage IV disease.

We will analyse women undergoing UDS and IDS in separate analyses (see above).

\section{Sensitivity analysis}

We will perform a sensitivity analysis that restricts the analyses to studies we judged to be at an overall low risk of bias.

We will also perform a sensitivity analysis to distinguish between studies that include $0 \mathrm{~cm}$ in residual disease categories of $<1 \mathrm{~cm}$ and those that do not. This may be applicable to comparisons involving $R D<1 \mathrm{~cm}$, with the exception of $R D<1 \mathrm{~cm}$ versus $R D=0$ $\mathrm{cm}$.

\section{Summary of findings and assessment of the certainty of the evidence}

Guidance on the use of GRADE for prognostic factor studies has not yet been published (Foroutan 2020; GRADE Working Group 2004), but we will attempt to appraise the quality and certainty of the evidence where possible. We will construct summary of findings tables to present the results of outcomes in the review for the main comparisons involving prognostic factor thresholds of $0 \mathrm{~cm},<1 \mathrm{~cm}$ and $>1 \mathrm{~cm}$. We will use the GRADE system to rank the certainty of the evidence (Foroutan 2020; GRADE Working Group 2004). Two review authors $(\mathrm{AB}, \mathrm{SH})$ will independently grade the evidence and resolve differences by discussion or by involving a third review author (PK). We will base our judgements on the strength of the body of evidence based on the domains presented in Appendix 3. Where the evidence is based on single studies, or where there is no evidence on a specific outcome for comparisons, we will include the outcome in the summary of findings table and will grade or explain in a narrative account accordingly. We will give the rationale for each judgement in the table footnotes. We will interpret the results of the review in light of this graded evidence. An example table is presented in Appendix 4.

\section{ACKN OWLEDGEMENTS}

We thank Jo Morrison, Tracey Harrison and Gail Quinn from Cochrane Gynaecological, Neuro-oncology and Orphan Cancers (GNOC) for their advice support and contribution to the editorial process. We thank Nicole Skoetz from the Prognostic Methods Group for her valuable input. We also thank Jo Platt, Information Manager for GNOC for designing the search strategies.

This project was supported by the National Institute for Health Research (NIHR), via Cochrane infrastructure funding to the Cochrane Gynaecological, Neuro-oncology and Orphan Cancers Group. The views and opinions expressed therein are those of the review authors and do not necessarily reflect those of the Systematic Reviews Programme, NIHR, NHS, or the Department of Health.

The authors and CNOC team are grateful to Sahar Salehi, Jennifer Hare and Philipp Harter for very helpful peer review comments. 


\section{R E F E R E N C E S}

\section{Additional references}

\section{Aletti 2007}

Aletti GD, Dowdy SC, Podratz KC, Cliby WA. Relationship among surgical complexity, short-term morbidity, and overall survival in primary surgery for advanced ovarian cancer. American Journal of Obstetrics and Gynecology 2007;197(6):676.

\section{Ang 2011}

Ang C, Chan KK L, Bryant A, Naik R, Dickinson HO. Ultraradical (extensive) surgery versus standard surgery for the primary cytoreduction of advanced epithelial ovarian cancer. Cochrane Database of Systematic Reviews 2011, Issue 4. Art. No: CD007697. [DOI: 10.1002/14651858.CD007697.pub2]

\section{Berek 2018}

Berek JS, Kehoe ST, Kumar L, Friedlander M. Cancer of the ovary, fallopian tube, and peritoneum. International Journal of Gynaecology and Obstetrics 2018;143:59-78.

\section{BGCS 2017}

Fotopoulou C, Hall M, Cruickshank D, Gabra H, Ganesan R, Hughes C, et al. British Gynaecological Cancer Society (BGCS) Epithelial Ovarian/Fallopian Tube/Primary Peritoneal Cancer Guidelines: Recommendations for Practice; 2017. Available at: www.bgcs.org.uk/wp-content/uploads/2019/05/BGCSGuidelines-Ovarian-Guidelines-2017.pdf.

\section{Bookman 2009}

Bookman MA, Brady MF, McGuire WP, Harper PG, Alberts DS, Friedlander $\mathrm{M}$, et al. Evaluation of new platinum-based treatment regimens in advanced-stage ovarian cancer: a Phase III Trial of the Gynecologic Cancer Intergroup. Journal of Clinical Oncology 2009;27(9):1419-25.

\section{Borley 2012}

Borley J, Wilhelm-Benartzi C, Brown R, Ghaem-Maghami S. Does tumour biology determine surgical success in the treatment of epithelial ovarian cancer? A systematic literature review. British Journal of Cancer 2012;107(7):1069-74.

\section{Bristow 2002}

Bristow RE, Tomacruz RS, Armstrong DK, Trimble EL, Montz FJ. Survival effect of maximal cytoreductive surgery for advanced ovarian carcinoma during the platinum era: a meta-analysis. Journal of Clinical Oncology 2002;20(5):1248-59.

\section{Bristow 2009}

Bristow RE, Zahurak ML, Diaz-Montes TP, Giuntoli RL, Armstrong DK. Impact of surgeon and hospital ovarian cancer surgical case volume on in hospital mortality and related shortterm outcomes. Gynecologic Oncology 2009;115(3):334e8.

\section{Chang 2013}

Chang SJ, Hodeib M, Chang J, Bristow RE. Survival impact of complete cytoreduction to no gross residual disease for advanced-stage ovarian cancer: a meta-analysis. Gynecologic Oncology 2013;130(3):493-8.

\section{Coleridge 2021}

Coleridge SL, Bryant A, Kehoe S, Morrison J. Neoadjuvant chemotherapy before surgery versus surgery followed by chemotherapy for initial treatment in advanced ovarian epithelial cancer. Cochrane Database of Systematic Reviews 2021, Issue 7. Art. No: CD005343. [DOI: 10.1002/14651858.CD005343]

\section{Colombo 2019}

Colombo N, Sessa C, du Bois A, Ledermann J, McCluggage WG, McNeish I, et al. ESMO-ESGO consensus conference recommendations on ovarian cancer: pathology and molecular biology, early and advanced stages, borderline tumours and recurrent disease. Annals of Oncology 2019;30(5):672-705.

\section{Covens 2000}

Covens AL. A critique of surgical cytoreduction in advanced ovarian cancer. Gynecologic Oncology 2000;78(3):269-74.

\section{Debray 2018}

Debray TP, Moons KG, Riley RD. Detecting small-study effects and funnel plot asymmetry in meta-analysis of survival data: a comparison of new and existing tests. Research Synthesis Methods 2018;9(1):41-50.

\section{Deeks 2001}

Deeks JJ, Altman DG, Bradburn MJ. Statistical methods for examining heterogeneity and combining results from several studies in meta-analysis. In: Egger M, Davey Smith G, Altman DG, editors(s). Systematic Reviews in Health Care: Meta-Analysis in Context. 2nd edition. London: BMJ Publication Group, 2001.

\section{DerSimonian 1986}

DerSimonian R, Laird N. Meta-analysis in clinical trials. Controlled Clinical Trials 1986;7:177-88.

\section{du Bois 2009}

du Bois A, Reuss A, Pujade-Lauraine E, Harter P, Ray-Coquard I, Pfisterer J. Role of surgical outcome as prognostic factor in advanced epithelial ovarian cancer: a combined exploratory analysis of 3 prospectively randomized phase 3 multicenter trials: by the Arbeitsgemeinschaft Gynaekologische Onkologie Studiengruppe Ovarialkarzinom (AGO-OVAR) and the Groupe d'Investigateurs Nationaux Pour les Etudes des Cancers de l'Ovaire (GINECO). Cancer 2009;115(6):1234-44.

\section{Elzarkaa 2018}

Elzarkaa AA, Shaalan W, Elemam D, Mansour H, Melis M, Malik E, et al. Peritoneal cancer index as a predictor of survival in advanced stage serous epithelial ovarian cancer: a prospective study. Journal of Gynecologic Oncology 2018;29(4):e47.

\section{EUROCARE 2015}

Sant M, Chirlaque Lopez MD, Agresti R, Sánchez Pérez MJ, Holleczek B, Bielska Lasota M, et al, EUROCARE-5 Working Group. Survival of women with cancers of breast and genital organs in Europe 1999-2007: Results of the EUROCARE-5 study. European Journal of Cancer 2015;51(15):2191-2205. 


\section{Fader 2007}

Fader AN, Rose PG. Role of surgery in ovarian carcinoma. Journal of Clinical Oncology 2007;25(20):2873-83.

\section{Fagotti 2020}

Fagotti A, Ferrandina MG, Vizzielli G, Pasciuto T, Fanfani F, Gallotta V, et al. Randomized trial of primary debulking surgery versus neoadjuvant chemotherapy for advanced epithelial ovarian cancer (SCORPION-NCT01461850). International Journal of Gynecological Cancer 2020;30(11):1657-1664. [PMID: 10.1136/ ijgc-2020- 001640]

\section{Falconer 2020}

Falconer H, Joneborg U, Krawiec K, Palsdottir K, Bottai M, Salehi S. Ultra-radical upfront surgery does not improve survival in women with advanced epithelial ovarian cancer; a natural experiment in a complete population. Gynecologic Oncology 2020;159(1):58-65.

\section{Foroutan 2020}

Foroutan F, Guyatt G, Zuk V, Vandvik PO, Alba AC, Mustafa R, et al. GRADE Guidelines 28: Use of GRADE for the assessment of evidence about prognostic factors: rating certainty in identification of groups of patients with different absolute risks. Journal of Clinical Epidemiology 2020;121:62-70. [DOI: 10.1016/ j.jclinepi.2019.12.023] [PMID: 31982539]

\section{Girling 1996}

Girling JC, Soutter WP. Cytoreductive surgery in the primary management of advanced epithelial ovarian carcinoma: a topic for debate. International Journal of Gynecological Cancer 1996;1:81-4.

\section{GLOBOCAN 2018}

Bray F, Ferlay J, Soerjomataram I, Siegel RL, Torre LA, Jemal, A. Global cancer statistics 2018: GLOBOCAN estimates of incidence and mortality worldwide for 36 cancers in 185 countries. CA: A Cancer Journal for Clinicians 2018;68:394-424. [DOI: 10.3322/ caac.21492]

\section{Grabowski 2016}

Grabowski JP, Harter P, Heitz F et al. Operability and chemotherapy responsiveness in advanced low-grade serous ovarian cancer. An analysis of the AGO Study Group metadatabase. Gynecologic Oncology 2016;140(3):457-62.

\section{GRADE Working Group 2004}

GRADE Working Group. Grading quality of evidence and strength of recommendations. BMJ 2004;328:1490-4.

\section{Greggi 2016}

Greggi S, Falcone F, Carputo R, et al. Primary surgical cytoreduction in advanced ovarian cancer: an outcome analysis within the MITO (Multicentre Italian Trials in Ovarian Cancer and Gynecologic Malignancies) Group. Gynecologic Oncology 2016;140(3):425-9.

\section{Griffiths 1975}

Griffiths CT. Surgical resection of tumor bulk in the primary treatment of ovarian carcinoma. Journal of the National Cancer Institute Monographs 1975;42:1014.

\section{Guyatt 2008}

Guyatt GH, Oxman AD, Vist GE, Kunz R, Falck-Ytter Y, AlonsoCoello $P$, et al. GRADE: an emerging consensus on rating quality of evidence and strength of recommendations. $B M J$ 2008;336(7650):924-6.

\section{Higgins 2003}

Higgins JPT, Thompson SG, Deeks JJ, Altman DG. Measuring inconsistency in meta-analyses. BMJ 2003;327:557-60.

\section{Hoskins 1994}

Hoskins WJ, McGuire WP, Brady MF, Homesley HD, Creasman WT, Berman M, et al. The effect of diameter of largest residual disease on survival after primary cytoreductive surgery in patients with suboptimal residual epithelial ovarian carcinoma: a Gynecologic Oncology Group study. American Journal of Obstetrics and Gynecology 1994;170:974-80.

\section{Hunter 1992}

Hunter RW, Alexander ND, Soutter WP. Meta-analysis of surgery in advanced ovarian carcinoma: Is maximum cytoreductive surgery an independent determinant of prognosis? American Journal of Obstetrics and Gynecology 1992;166:504-11.

\section{Jemal 2017}

Jemal A, Ward E, Johnson C, Cronin K, Ma J, Ryerson B, et al. Annual Report to the Nation on the Status of Cancer, 1975-2014, Featuring Survival Cancer Statistics. Journal of the National Cancer Institute 2017;109:djx030. [DOI: 10.1093/jnci/djx030]

\section{Kehoe 2015}

Kehoe S, Hook J, Nankivell M, et al. Primary chemotherapy versus primary surgery for newly diagnosed advanced ovarian cancer (CHORUS): an open-label, randomised, controlled, noninferiority trial. Lancet 2015;386(9990):249-57.

\section{Kumar 2017}

Kumar A, Langstraat CL, DeJong SR, McGree ME, BakkumGamez JN, Weaver AL, et al. Functional not chronologic age: frailty index predicts outcomes in advanced ovarian cancer. Gynecologic Oncology 2017;147(1):104-9.

\section{Kurman 2010}

Kurman RJ, Shih leM. The origin and pathogenesis of epithelial ovarian cancer: a proposed unifying theory. American Journal of Surgical Pathology 2010;34(3):433-43.

\section{Kurman 2011}

Kurman RJ, Shih leM. Molecular pathogenesis and extra ovarian origin of epithelial ovarian cancer-shifting the paradigm. Human Pathology 2011;42(7):918-31.

\section{Kurman 2014}

Kurman RJ, Carcangiu ML, Herrington CS. WHO classification of tumours of female reproductive organs. 4th edition. Lyon: WHO Press, 2014.

\section{Markar 2016}

Makar AP, Tropé CG, Tummers P, Denys H, Vandecasteele K. Advanced ovarian cancer: primary or interval debulking? Five categories of patients in view of the results of randomized trials

Impact of residual disease as a prognostic factor for survival in women with advanced epithelial ovarian cancer after primary surgery 
and tumor biology: primary debulking surgery and interval debulking surgery for advanced ovarian cancer. Oncologist 2016;21(6):745-54.

\section{Moons 2014}

Moons KG, de Groot JA, Bouwmeester W, Vergouwe Y, Mallett S, Altman DG, et al. Critical appraisal and data extraction for systematic reviews of prediction modelling studies: the CHARMS checklist. PLOS Medicine 2014;11(10):e1001744.

\section{Napoletano 2010}

Napoletano C, Bellati F, Landi R, Pauselli S, Marchetti C, Visconti V, et al. Ovarian cancer cytoreduction induces changes in T cell population subsets reducing immunosuppression. Journal of Cellular and Molecular Medicine 2010;14(12):2748-59.

\section{National Comprehensive Cancer Network 2020}

National Comprehensive Cancer Network. NCCN Clinical Practice Guidelines in Oncology, Ovarian Cancer Including Fallopian Tube Cancer and Primary Peritoneal Cancer. Available at: www.nccn.org/professionals/physician_gls/pdf/ovarian.pdf 2020.

\section{Parmar 1998}

Parmar MK, Torri V, Stewart L. Extracting summary statistics to perform meta-analyses of the published literature for survival endpoints. Statistics in Medicine 1998;17(24):2815-34.

\section{Perets 2016}

Perets R, Drapkin R. It's Totally Tubular. Riding The New Wave of Ovarian Cancer Research. Cancer Research 2016;76(1):10-7.

\section{Redman 1994}

Redman CW, Warwick J, Luesley DM, Varma R, Lawton FG, Blackledge GR. Intervention debulking surgery in advanced epithelial ovarian cancer. British Journal of Obstetrics and Gynaecology 1994;101:142-6.

\section{Reuss 2019}

Reuss A, du Bois A, Harter P, Fotopoulou C, Sehouli J, Aletti G, Guyon F, et al. TRUST: trial of radical upfront surgical therapy in advanced ovarian cancer (ENGOT ov33/AGO-OVAR OP7). International Journal of Gynecologic Cancer 2019;29(8):1327-31.

\section{Review Manager 2020 [Computer program]}

Nordic Cochrane Centre, The Cochrane Collaboration Review Manager 5 (RevMan 5). Version 5.4. Copenhagen: Nordic Cochrane Centre, The Cochrane Collaboration, 2020.

\section{Riley 2019}

Riley RD, Moons KG, Snell KI, Ensor J, Hooft L, Altman DG et al. A guide to systematic review and meta-analysis of prognostic factor studies. BMJ 2019;364:k4597.

\section{Rose 2004}

Rose PG, Nerenstone S, Brady MF, Clarke-Pearson D, Olt G, Rubin SC, et al. Secondary surgical cytoreduction for advanced ovarian carcinoma. New England Journal of Medicine 2004;351:2489-97.

\section{Shafi 2018}

Shafi M, Bolton H, Gajjar K, editor(s). Gynaecological Oncology for the MRCOG. Cambridge: Cambridge University Press, 2018. [DOI: $10.1017 / 9781316986844]$

\section{Sterne 2011}

Sterne JA, Egger M, Moher D, editor(s). Chapter 10: Addressing reporting biases. In: Higgins JP, Green S, editor(s). Cochrane Handbook for Systematic Reviews of Interventions Version 5.1.0 (updated March 2011). The Cochrane Collaboration, 2011. Available from training.cochrane.org/handbook/archive/v5.1.

\section{Stuart 2011}

Stuart GC, Kitchener H, Bacon M, et al. Gynecologic Cancer InterGroup (GCIG) consensus statement on clinical trials in ovarian cancer: report from the Fourth Ovarian Cancer Consensus Conference. International Journal of Gynecological Cancer 2011;21(4):750-5.

\section{Sørensen 2019}

Sørensen SM, Schnack TH, Høgdall C. Impact of residual disease on overall survival in women with Federation of Gynecology and Obstetrics stage IIIB-IIIC vs stage IV epithelial ovarian cancer after primary surgery. Acta Obstetricia et Gynecologica Scandinavica 2019;98(1):34-43.

\section{Tangjitgamol 2016}

Tangjitgamol S, Manusirivithaya S, Laopaiboon M, Lumbiganon P, Bryant A. Interval debulking surgery for advanced epithelial ovarian cancer. Cochrane Database of Systematic Reviews 2016, Issue 1. Art. No: CD006014. [DOI: 10.1002/14651858.CD006014]

\section{Torre 2018}

Torre LA, Trabert B, DeSantis CE, Miller K, Samimi G, Runowicz C, et al. Ovarian cancer statistics. CA: a Cancer Journal for Clinicians 2018;68(4):284-96.

\section{Van der Burg 1995}

van der Burg ME, van Lent M, Buyse M, Kobierska A, Colombo N, Favalli $G$, et al. The effect of debulking surgery after induction chemotherapy on the prognosis in advanced epithelial ovarian cancer. Gynecological Cancer Cooperative Group of the European Organization for Research and Treatment of Cancer. New England Journal of Medicine 1995;332(10):629-34.

\section{Vergote 2010}

Vergote I, Tropé CG, Amant F, et al. Neoadjuvant chemotherapy or primary surgery in stage IIIC or IV ovarian cancer. New England Journal of Medicine 2010;363(10):943-53.

\section{Vergote 2013}

Vergote I, du Bois A, Amant F, Heitz F, Leunen K, Harter P. Neoadjuvant chemotherapy in advanced ovarian cancer: on what do we agree and disagree? Gynecologic Oncology 2013;128:6-11.

\section{Vergote 2016}

Vergote I, Vlayen J, Heus P, Hoogendam JP, Damen JA, Van de Wetering FT, et al. Ovarian cancer: diagnosis, treatment and follow-up; KCE Report 28Cs; 2016. Available at: kce.fgov.be/ 
sites/default/files/atoms/files/KCE_268Cs_Ovarian_cancer_ summary.pdf.

\section{Vergote 2018}

Vergote I, Coens C, Nankivell M, Kristensen GB, Parmar MKB, Ehlen T, et al. Neoadjuvant chemotherapy versus debulking surgery in advanced tubo-ovarian cancers: pooled analysis of individual patient data from the EORTC 55971 and CHORUS trials. Lancet Oncology 2018 [published correction appears in Lancet Oncology 2019 Jan;20(1):e10];19(12):1680-7.

\section{Webb 2017}

Webb PM, Jordan SJ. Epidemiology of epithelial ovarian cancer, best practice \& research. Clinical Obstetrics \& Gynaecology 2017;41:3-14.

\section{Whiting 2011}

Whiting PF, Rutjes AW, Westwood ME, Mallett S, Deeks, JJ, Reitsma JB, et al. QUADAS-2: a revised tool for the quality assessment of diagnostic accuracy studies. Annals of Internal Medicine 2011;155(8):529-36.

\section{AP PEN DICES}

\section{Appendix 1. MEDLINE search strategy}

1. exp Ovarian Neoplasms/

2. (ovar* adj5 cancer $\left.{ }^{\star}\right) \cdot \mathrm{mp}$.

3. (ovar ${ }^{\star}$ adj5 neoplas*).mp.

4. (ovar* ${ }^{\star}$ adj5 carcinom*).mp.

5. (ovar ${ }^{\star}$ adj5 malignan $\left.{ }^{\star}\right) \cdot m p$.

6. $\left(\right.$ ovar $^{\star}$ adj5 tumor $\left.{ }^{\star}\right) \cdot \mathrm{mp}$.

7. $\left(\right.$ ovar $^{\star}$ adj5 tumour $\left.{ }^{\star}\right) \cdot m p$.

8. 1 or 2 or 3 or 4 or 5 or 6 or 7

9. exp Surgical Procedures, Operative/

10.surg*.mp.

11."surgery".fs.

12.9 or 10 or 11

13. debulk*.mp.

14. cytoreduc ${ }^{\star} . \mathrm{mp}$.

15.13 or 14

16.8 and 12 and 15

17."randomized controlled trial".pt.

18."controlled clinical trial".pt.

19.random*.mp.

20.trial* ${ }^{*} . \mathrm{mp}$.

21.group ${ }^{\star} . \mathrm{mp}$.

22.exp Cohort Studies/

23. cohort*.mp.

24.series.mp.

25.17 or 18 or 19 or 20 or 21 or 22 or 23 or 24

26.16 and 25

27.Animals/

28. Humans/

29.27 not ( 27 and 28$)$

30.26 not 29

\section{Wimberger 2010}

Wimberger P, Wehling M, Lehmann N, Kimmig R, Schmalfeldt B, Burges A, et al. Influence of residual tumor on outcome in ovarian cancer patients with FIGO stage IV disease: an exploratory analysis of the AGO-OVAR (Arbeitsgemeinschaft Gynaekologische Onkologie Ovarian Cancer Study Group). Annals of Surgical Oncology 2010;17(6):1642-8.

\section{Wolff 2019}

Wolff RF, Moons KGM, Riley RD, Whiting PF, Westwood M, Collins GS, et al. PROBAST: a tool to assess the risk of bias and applicability of prediction model studies. Annals of Internal Medicine 2019;170(1):51-8.

\section{Woo 2012}

Woo YL, Kyrgiou M, Bryant A, Everett T, Dickinson HO. Centralisation of services for gynaecological cancer. Cochrane Database of Systematic Reviews 2012, Issue 3. Art. No: CD007945. [DOI: 10.1002/14651858.CD007945.pub2] 
key: $\mathrm{mp}=$ title, original title, abstract, name of substance word, subject heading word, $\mathrm{fs}=$ floating subheading, $\mathrm{pt}=$ publication type

\section{Appendix 2. Risk of bias and applicability assessment}

Risk of bias and applicability assessment tool to assess risk of bias and applicability of prognostic factor studies (Riley 2019). Signalling questions and risk of bias ratings are listed in bullet points.

\section{Domain 1: participant selection}

\section{Risk of bias}

- Adequate participation in the study by eligible persons

- Description of the target population or population of interest

- Description of the baseline study sample

- Adequate description of the sampling frame and recruitment

- Adequate description of the period and place of recruitment

- Adequate description of inclusion and exclusion criteria

\section{Risk of bias ratings}

- High: the relationship between the PF and outcome is very likely to be different for participants and eligible non-participants.

- Moderate: the relationship between the PF and outcome may be different for participants and eligible non-participants.

- Low: the relationship between the PF and outcome is unlikely to be different for participants and eligible non-participants.

\section{Applicability}

Are there concerns that the included women do not match the review question?

\section{Domain 2: study attrition}

\section{Risk of bias}

- Adequate response rate for study participants

- Description of attempts to collect information on participants who dropped out

- Reasons for loss to follow-up are provided

- Adequate description of participants lost to follow-up

- There are no important differences between participants who completed the study and those who did not

\section{Risk of bias ratings}

- High: the relationship between the PF and outcome is very likely to be different for completing and non-completing participants.

- Moderate: the relationship between the PF and outcome may be different for completing and non-completing participants.

- Low: the relationship between the PF and outcome is unlikely to be different for completing and non-completing participants.

\section{Domain 3: prognostic factor measurement}

\section{Risk of bias}

- A clear definition or description of the PF is provided.

- Method of PF measurement is adequately valid and reliable.

- Continuous variables are reported or appropriate cutpoints are used.

- The method and setting of measurement of PF is the same for all study participants.

- Adequate proportion of the study sample has complete data for the PF.

- Appropriate methods of imputation are used for missing PF data.

\section{Risk of bias ratings}

- High: the measurement of the PF is very likely to be different for different levels of the outcome of interest.

- Moderate: the measurement of the PF may be different for different levels of the outcome of interest.

- Low: the measurement of the PF is unlikely to be different for different levels of the outcome of interest.

\section{Applicability}

Are there concerns that residual disease, the way that it is measured, or the way that it is interpreted, differ from the review question?

Impact of residual disease as a prognostic factor for survival in women with advanced epithelial ovarian cancer after primary surgery 


\section{Domain 4: outcome measurement}

\section{Risk of bias}

- A clear definition of the outcome is provided.

- Method of outcome measurement used is adequately valid and reliable.

- The method and setting of outcome measurement is the same for all study participants.

\section{Risk of bias ratings}

- High: the measurement of the outcome is very likely to be different related to the baseline level of the PF.

- Moderate: the measurement of the outcome may be different related to the baseline level of the PF.

- Low: the measurement of the outcome is unlikely to be different related to the baseline level of the PF.

\section{Applicability}

Are there concerns that outcome does not match the review question or that follow-up was not of sufficient duration?

\section{Domain 5: adjustment for other prognostic factors}

\section{Risk of bias}

- All other important PFs are measured.

- Clear definitions of the important PFs measured are provided.

- Measurement of all important PFs is adequately valid and reliable.

- The method and setting of PF measurement are the same for all study participants.

- Appropriate methods are used to deal with missing values of PFs, such as multiple imputation.

- Important PFs are accounted for in the study design.

- Important PFs are accounted for in the analysis.

\section{Risk of bias ratings}

- High: the observed effect of the PF on the outcome is very likely to be distorted by another factor related to PF and outcome.

- Moderate: the observed effect of the PF on outcome may be distorted by another factor related to PF and outcome.

- Low: the observed effect of the PF on outcome is unlikely to be distorted by another factor related to PF and outcome.

\section{Applicability}

Did the prognostic factors adjusted for match the review question?

\section{Domain 6: statistical analysis and reporting}

\section{Risk of bias}

- There is sufficient presentation of data to assess the adequacy of the analytic strategy.

- Strategy for model building is appropriate and is based on a conceptual framework or model.

- The selected statistical model is adequate for the design of the study.

- There is no selective reporting of results.

\section{Risk of bias ratings}

- High: the reported results are very likely to be spurious or biased related to analysis or reporting.

- Moderate: the reported results may be spurious or biased related to analysis or reporting.

- Low: the reported results are unlikely to be spurious or biased related to analysis or reporting.

$\mathrm{PF}=$ prognostic factor

\section{Appendix 3. Domains to be considered when judging the strength of the body of evidence}

We will consider the following domains when we assess the strength of the body of evidence, based on the GRADE approach (Guyatt 2008).

- Risk of bias: based on results of 'Risk of bias' assessments, we will downgrade confidence in the evidence base if most evidence is from studies that we judge to be at high risk of bias.

- Indirectness: we will downgrade confidence in the evidence base if we have concerns that the study sample, the prognostic factor, the outcome, or the other factors in the models in the primary studies do not reflect the review question. 
- Inconsistency: we will downgrade confidence in the evidence base if there is unexplained heterogeneity or variability in results across studies.

- Imprecision: we will downgrade confidence in the evidence base if the estimate of the effect size from a meta-analysis is not precise or, if no meta-analysis is performed, if the estimate of the size of effect from individual studies is not precise.

- Publication bias: studies showing no association are likely to be unpublished, unless part of a larger study that specifically aimed to compare tests. We will downgrade our confidence in the evidence base if we have reason to suspect publication bias from our assessments of reporting bias.

- Size of effect: we will upgrade our confidence in the evidence base if the size of effect is moderate or large. If a meta-analysis is not possible, we will upgrade if the size of effect is moderate or large for most included studies.

\section{Appendix 4. Summary of findings table}

\section{Comparison of different residual disease (RD) thresholds after primary surgery in women with advanced epithelial ovarian} cancer

\section{Population:}

Setting:

\begin{tabular}{|c|c|c|c|c|c|c|}
\hline \multirow[t]{2}{*}{ Outcomes } & \multicolumn{2}{|c|}{$\begin{array}{l}\text { Anticipated absolute effects }{ }^{\star} \text { ( } 95 \% \\
\mathrm{CI})\end{array}$} & \multirow{2}{*}{$\begin{array}{l}\text { Relative } \\
\text { effect (HR) } \\
(95 \% \mathrm{Cl})\end{array}$} & \multirow{2}{*}{$\begin{array}{l}\text { No of Par- } \\
\text { ticipants } \\
\text { (studies) }\end{array}$} & \multirow[t]{2}{*}{$\begin{array}{l}\text { Quality of the evidence } \\
\text { (GRADE) }\end{array}$} & \multirow[t]{2}{*}{ Comments } \\
\hline & $\begin{array}{l}\text { Risk with sub- } \\
\text { optimal RD }\end{array}$ & $\begin{array}{l}\text { Risk with optimal } \\
\text { RD }\end{array}$ & & & & \\
\hline \multirow{4}{*}{$\begin{array}{l}\text { Overall } \\
\text { survival } \\
\text { Follow-up: }\end{array}$} & \multicolumn{2}{|l|}{ FIGO stage III } & \multirow[t]{4}{*}{$\mathrm{HR}()$} & \multirow[t]{4}{*}{$\mathrm{n}(\mathrm{n})$} & \multirow{4}{*}{$\begin{array}{l}\text { [To be deleted as appropriate] } \\
\oplus \odot \odot \odot \\
\text { very low }\end{array}$} & \\
\hline & n per 1000 & $\begin{array}{l}\mathrm{n} \text { per } 1000 \\
(\mathrm{x} \text { to } \mathrm{y})\end{array}$ & & & & \\
\hline & \multicolumn{2}{|l|}{ FIGO stage IV } & & & & \\
\hline & n per 1000 & $\begin{array}{l}\mathrm{n} \text { per } 1000 \\
(\mathrm{x} \text { to } \mathrm{y})\end{array}$ & & & & \\
\hline \multirow{5}{*}{$\begin{array}{l}\text { Progres- } \\
\text { sion-free } \\
\text { survival } \\
\text { Follow-up: }\end{array}$} & \multicolumn{2}{|l|}{ FIGO stage III } & \multirow[t]{5}{*}{$\mathrm{HR}()$} & \multirow[t]{5}{*}{$n(n)$} & \multirow[t]{2}{*}{ [To be deleted as appropriate] } & \\
\hline & n per 1000 & $\begin{array}{l}\text { n per } 1000 \\
(x \text { to } y)\end{array}$ & & & & \\
\hline & \multicolumn{2}{|l|}{ FIGO stage IV } & & & $\begin{array}{l}\oplus \oplus \ominus \ominus \\
\text { low }\end{array}$ & \\
\hline & n per 1000 & $\begin{array}{l}\text { n per } 1000 \\
\text { (x to } y)\end{array}$ & & & $\begin{array}{l}\oplus \oplus \oplus \ominus \\
\text { moderate }\end{array}$ & \\
\hline & & & & & $\begin{array}{l}\oplus \oplus \oplus \oplus \\
\text { high }\end{array}$ & \\
\hline
\end{tabular}

*The survival in the optimal RD group (and its 95\% confidence interval) is based on the assumed survival in the suboptimal RD group.

CI: Confidence interval; FIGO: International Federation of Gynecology and Obstetrics; HR: Hazard Ratio; RD: Residual disease

GRADE Working Group grades of evidence

High quality: we are very confident that the true effect lies close to that of the estimate of the effect.

Impact of residual disease as a prognostic factor for survival in women with advanced epithelial ovarian cancer after primary surgery 
(Continued)

Moderate quality: we are moderately confident in the effect estimate. The true effect is likely to be close to the estimate of the effect, but there is a possibility that it is substantially different.

Low quality: our confidence in the effect estimate is limited. The true effect may be substantially different from the estimate of the effect.

Very low quality: we have very little confidence in the effect estimate. The true effect is likely to be substantially different from the estimate of effect.

\section{CONTRIBUTIONS OF AUTHORS}

Andrew Bryant was lead author of the protocol and drafted methodological sections and addressed peer review comments. Shaun Hiu and Patience Kunonga contributed to the draft of the protocol. Dawn Craig and Luke Vale contributed to the draft of the protocol and added methodological expertise. Ketankumar Gajjar drafted clinical sections of the protocol and addressed peer review comments. Brett WinterRoach and Ahmed Elattar drafted clinical sections of the protocol. Raj Naik drafted clinical sections of the protocol and was lead clinical expert.

\section{DECLARATIONS OF INTEREST}

$A B$ : None known

SH: None known

PK: None known

KG: None known

DC: None known

LV: None known

BAW-R: None known

AE: None known

RN: None Known

\section{SOURCES OF SUPPORT}

\section{Internal sources}

- No sources of support provided

\section{External sources}

- No sources of support provided 